\title{
Dinâmica temporal da podridão cinzenta (Botrytis cinerea) na videira Cabernet Franc em função do aumento da carga de gemas
}

\author{
Temporal dynamics of botrytis bunch rot (Botrytis cinerea) in Cabernet Franc grapevine as a \\ function of increased bud load
}

Dinámica temporal de la podredumbre gris (Botrytis cinerea) en la vid Cabernet Franc en función del aumento de la carga de yemas

Recebido: 11/07/2021 | Revisado: 16/07/2021 | Aceito: 16/07/2021 | Publicado: 25/07/2021

\author{
Douglas André Wurz \\ ORCID: https://orcid.org/0000-0001-6109-9858 \\ Instituto Federal de Santa Catarina, Brasil \\ E-mail: douglas.wurz@ifsc.edu.br \\ Alberto Fontanella Brighenti \\ ORCID: https://orcid.org/0000-0002-0831-4640 \\ Universidade Federal de Santa Catarina, Brasil \\ E-mail: alberto.brighenti@ufsc.br \\ Bruno Farias Bonin \\ ORCID: https://orcid.org/0000-0001-6983-2970 \\ Universidade Federal do Paraná, Brasil \\ E-mail:brunobonin@hotmail.com \\ Ricardo Allebrandt \\ ORCID: https://orcid.org/0000-0001-8222-6254 \\ Centro Universitário Dinâmica das Cataratas, Brasil \\ E-mail: ricardoufsc@gmail.com \\ Betina Pereira de Bem \\ ORCID: https://orcid.org/0000-0003-3783-7018 \\ Vinícola Quinta da Neve, Brasil \\ E-mail: betadebem@yahoo.com.br \\ Adrielen Tamiris Canossa \\ ORCID: https://orcid.org/0000-0002-4867-3293 \\ Instituto Federal de Santa Catarina, Brasil \\ E-mail: adrielencanossa@yahoo.com.br \\ Leo Rufato \\ ORCID: https://orcid.org/0000-0001-9545-7035 \\ Universidade do Estado de Santa Catarina, Brasil \\ E-mail: leorufato@udesc.br
}

\begin{abstract}
Resumo
O aumento do dossel vegetativo e o adensamento de cachos na planta ocasionado pelo maior número de gemas planta ${ }^{1}$ pode criar um microclima favorável para a ocorrência de podridão cinzenta. O presente trabalho teve como objetivo avaliar o efeito do aumento de carga de gemas planta ${ }^{-1}$, nas variáveis epidemiológicas de podridão cinzenta (Botrytis cinerea) na videira Cabernet Franc cultivada em região de altitude de Santa Catarina. O presente trabalho foi conduzido durante safra 2016/2017, em um vinhedo comercial, localizado no munícipio de São Joaquim. Os tratamentos consistiram em quatro diferentes níveis de poda: 15 gemas planta $^{-1}, 30$ gemas planta $^{-1}, 50$ gemas planta $^{-1}$, 75 gemas planta ${ }^{-1}$. Avaliou-se: incidência máxima, severidade máxima, AACPID, AACPSD, TAMID, TAMSD e IAS. Observou-se efeito da carga de gemas planta ${ }^{-1}$ nas variáveis epidemiológicas da podridão cinzenta nos cachos da videira Cabernet Franc. $\mathrm{O}$ aumento da carga de gemas resultou em aumento da incidência e da severidade da doença, AACPID e AACPSD, sendo os maiores observados em plantas submetidas a poda de inverno com carga de gemas superiores a 50 gemas planta ${ }^{-1}$. A adoção de elevado número de gemas planta ${ }^{-1}$, deve ser acompanhada de práticas de manejo integrado de doenças que visam reduzir os efeitos ocasionados pela ocorrência de podridão cinzenta.
\end{abstract}

Palavras-chave: Vitis vinifera L.; Podridão de cachos; Manejo integrado de doenças; Poda.

\section{Abstract}

The increase in the vegetative canopy and the thickening of bunches in the plant caused by the higher number of plant ${ }^{-}$ ${ }^{1}$ buds can create a favorable microclimate for the occurrence of Botrytis bunch rot. This study aimed to evaluate the effect of increasing plant-1 bud load on epidemiological variables of Botrytis bunch rot (Botrytis cinerea) in the Cabernet Franc vine cultivated in an altitude region of Santa Catarina State. The present work was carried out during 
the 2016/2017 harvest, in a commercial vineyard, located in the municipality of São Joaquim. The treatments consisted of four different pruning levels: 15 buds plant-1, 30 buds plant-1, 50 buds plant-1, 75 buds plant- 1 . It was evaluated: maximum incidence, maximum severity, AACPID, AACPSD, TAMID, TAMSD and IAS. It was observed an effect of plant-1 bud load on the epidemiological variables of Botrytis bunch rot in the Cabernet Franc grapevine bunches. The increase in bud load resulted in an increase in the incidence and severity of the disease, AACPID and AACPSD, the highest being observed in plants submitted to winter pruning with a bud load greater than 50 buds plant-1. The adoption of a high number of plant-1 buds must be accompanied by integrated disease management practices that aim to reduce the effects caused by the occurrence of Botrytis bunch rot.

Keywords: Vitis vinifera L.; Botrytis bunch rot; Integrated disease management; Pruning.

\section{Resumen}

El aumento del dosel vegetativo y el engrosamiento de los racimos en la planta causado por el mayor número de yemas de la planta pueden crear un microclima favorable para la aparición de la pudrición gris. Este estudio tuvo como objetivo evaluar el efecto del aumento de la carga de yemas de la planta sobre las variables epidemiológicas de la podredumbre gris (Botrytis cinerea) en la vid Cabernet Franc cultivada en una región altitudinal de Santa Catarina. El presente trabajo se realizó durante la vendimia 2016/2017, en un viñedo comercial, ubicado en el municipio de São Joaquim. Los tratamientos consistieron en cuatro niveles de poda diferentes: 15 yemas planta $^{-1}, 30$ yemas planta $^{-1}, 50$ yemas planta ${ }^{-1}, 75$ yemas planta ${ }^{-1}$. Se evaluó: máxima incidencia, máxima severidad, AACPID, AACPSD, TAMID, TAMSD e IAS. Se observó un efecto de la carga de yemas de la planta sobre las variables epidemiológicas de la pudrición gris en racimos de vid Cabernet Franc. El aumento en la carga de yemas resultó en un aumento en la incidencia y severidad de la enfermedad, AACPID y AACPSD, siendo el mayor observado en plantas sometidas a poda de invierno con una carga de yemas superior a 50 yemas planta ${ }^{-1}$ La adopción de una gran cantidad de yemas de la planta debe ir acompañada de prácticas integradas de manejo de enfermedades que tengan como objetivo reducir los efectos causados por la aparición de la pudrición gris.

Palabras clave: Vitis vinifera L.; Pudrición del racimo; Manejo integrado de enfermedades; Poda.

\section{Introdução}

A poda invernal é uma prática realizada anualmente em regiões de clima temperado, com o principal objetivo de regular a produção temporal da videira (Miele \& Mandelli, 2012). A definição da intensidade da poda pelo viticultor depende de vários fatores, mas, de toda maneira, procura-se harmonizar a produtividade do vinhedo com a qualidade da uva. Saliente-se que a produtividade do vinhedo se correlaciona positivamente com o número de gemas deixadas por planta, ainda que, em geral, essa relação não seja linear (Miele \& Rizzon, 2013).

Os processos adaptativos pelos quais as videiras respondem ao aumento do número de gemas, foram descritos, e incluem redução do crescimento vegetativo, redução da fertilidade de gemas, ramos mais curtos com entrenós mais curtos, maior produtividade e maior número de cachos por planta, além de apresentarem cachos mais longos e com bagas menores (Clingeleffer, 2009; Wurz et al., 2020a; Greven et al., 2014; Greven et al., 2015).

Pesquisas pioneiras realizadas na Nova Zelândia por Jackson et al. (1984) descreveram que o número de gemas variando de 40 a 150 por plantas, em cinco apresenta um comportamento curvilíneo, onde o aumento de 43 para 86 gemas por plantou dobrou a produção. Trabalho realizado por Greven et al. (2015), o aumento de 24 para 72 gemas por planta resultou em aumento da produtividade de 4,8 para 12,7 ton/ha.

No entanto, de acordo com Wurz et al. (2017a) e Wurz et al. (2020a), o aumento da carga de gemas da videira Sauvignon Blanc pode resultar em um dossel mais denso com aumento do número de cachos por planta, afetando a ocorrência doenças fúngicas. $\mathrm{O}$ aumento do dossel vegetativo e o adensamento de cachos na planta ocasionado pelo maior número de gemas planta-1, pode criar um microclima favorável para a ocorrência de podridão cinzenta (Botrytis cinerea). De acordo com Zoecklin et al. (1992) e Mossetti et al. (2016), o desenvolvimento de B. cinerea é fortemente influenciado pelo microclima na zona dos cachos, sendo influenciado pelo período de molhamento foliar.

A ocorrência da podridão cinzenta ou podridão de Botrytis causada pelo fungo Botryotinia fuckeliana (de Bary) Whetzel, forma sexuada de Botrytis cinerea Persoon ex Fries pode comprometer a produção e qualidade de frutos (Ellis, 1971). O fungo pode atacar quase todos os órgãos e se manifestar a partir da primavera, mas são os ataques aos cachos, durante a 
maturação que assumem maior gravidade. Os primeiros sintomas da doença nas bagas de uvas são a presença de pontuações ligeiramente claras, circulares de 2 a $3 \mathrm{~mm}$, que podem ser observadas 72 horas após a penetração do fungo nos tecidos, podendo deixar a polpa exposta. Depois que a infecção se estabeleceu, o fungo cresce através das rachaduras na casca das bagas e produz grande quantidade de esporos (Garrido \& Sonego, 2005).

Buscando compreender a influência da carga de gemas não ocorrência de podridão cinzenta, o presente trabalho teve como objetivo avaliar o efeito do aumento de carga de gemas planta1, nas variáveis epidemiológicas de podridão cinzenta (Botrytis cinerea) na videira Cabernet Franc cultivada em região de altitude de Santa Catarina.

\section{Metodologia}

O presente trabalho foi conduzido durante safra 2016/2017, em um vinhedo comercial, localizado no munícipio de São Joaquim, coordenadas ( $28^{\circ} 17^{\prime} 39^{\prime \prime}$ S e 49 $55^{\prime} 56^{\prime \prime}$ O), a 1230 metros de altitude acima do nível do mar. Utilizaram-se plantas a cultivar Cabernet Franc enxertadas sobre o porta-enxerto 'Paulsen 1103'. O delineamento experimental utilizado foi o de blocos ao acaso, com quatro blocos e dez plantas por repetição

Os vinhedos foram implantados em 2004, apresentando plantas com espaçamento 3,0 x 1,5m, em filas dispostas no sentido N-S, conduzidas em espaldeira, a 1,2m de altura e cobertas com tela de proteção anti-granizo. Os solos da região enquadram-se nas classes Cambissolo Húmico, Neossolo Litólico e Nitossolo Háplico, desenvolvidos a partir de rocha riodacito e basalto (SANTOS et al., 2018). O clima da região é classificado como Subtropical de altitude, 'Frio, Noites Frias e Úmido', Índice Heliotérmico de 1.714, precipitação pluvial média anual de $1.621 \mathrm{~mm}$ e a umidade relativa do ar média anual de $80 \%$ (Tonietto \& Carbonnau, 2004).

Os tratamentos consistiram em quatro diferentes níveis de poda: 15 gemas planta-1, 30 gemas planta-1, 50 gemas planta-1, 75 gemas planta-1. A poda foi realizada no dia 08 de Setembro de 2016. Deixou-se 8, 15, 25 esporões com duas gemas cada esporão para os tratamentos 15, 30 e 50 gemas planta-1, e para o tratamento 75 gemas planta-1, deixou-se 30 esporões com duas gemas, e duas varas com 8 gemas cada, sendo portanto, este tratamento podado no sistema de poda mista, caracterizado pela presença de esporões e varas.

O manejo da videira (poda, desbrote, condução, desfolha e colheita) foi realizado pelo grupo de Fruticultura do Centro de Ciências Agroveterinárias da Universidade do Estado de Santa Catarina, com o objetivo de obter maior controle experimental, sendo realizado de acordo com os padrões da empresa que cedeu os vinhedos para a realização do experimento.

A incidência de Botrytis cinerea foi obtida através de avaliação visual, sendo verificada a presença ou ausência de sintomas da doença. Sendo a incidência calculada pela porcentagem de cachos que apresentavam ao menos uma lesão em relação ao número total de cachos. Para a severidade de Botrytis cinerea, as avaliações iniciaram ao surgimento dos primeiros sintomas, em intervalos de 10 a 15 dias, sob condições de infecção natural. Foram demarcados 20 cachos/parcela, totalizando 80 cachos para cada carga de gemas, marcados aleatoriamente, e as avaliações foram realizadas através de escala diagramática proposta por Hill et al., (2010).

Com os dados obtidos da podridão cinzenta da videira foram plotadas curvas de progresso da incidência e da severidade, e a epidemia foi comparada em relação ao: início do aparecimento dos sintomas (IAS) (dias); tempo para atingir a máxima incidência e severidade da doença (TAMID e TAMSD) (dias); valor máximo da incidência (Imax) (\%) e severidade (Smax) (\%); área abaixo da curva de progresso da incidência (AACPI) e da severidade (AACPS). Para o cálculo da Área Abaixo da Curva de Progresso de Doença (AACPD) utilizou-se a fórmula: AACPD $=\Sigma((Y i+Y i+1) / 2)(\mathrm{ti}+1-\mathrm{ti})$, onde $\mathrm{Y}$ representa a intensidade (incidência e severidade) da doença, t o tempo e i o número de avaliações no tempo (Campbell \& Madden, 1990).

Os dados meteorológicos foram obtidos a partir de Estação Meteorológica Automática Telemétrica do Centro de 
Informações de Recursos Ambientais e de Hidrometeorologia de Santa Catarina (EPAGRI/CIRAM), localizada na Estação Experimental da EPAGRI em São Joaquim. As variáveis meteorológicas registradas foram: temperatura média do ar (o C) e precipitação pluviométrica $(\mathrm{mm})$ diária durante os meses de agosto de 2016 à Abril de 2017, correspondendo ao período vegetativo da videira na safra avaliada.

Os dados das médias de incidência da doença foram transformados pelo arco seno da raiz quadrada para normalização da distribuição estatística. As médias foram submetidas à análise de variância (ANOVA) e a detecção de diferenças significativas entre os tratamentos foi obtida através do teste Tukey a $5 \%$.

\section{Resultados e Discussão}

Ocorreram condições climáticas favoráveis ao desenvolvimento das principais doenças fúngicas da videira nos dois ciclos avaliados. No período de maior crescimento vegetativo do vinhedo (dezembro a março) no ciclo 2016/2017 a temperatura média foi de $17,3^{\circ} \mathrm{C}$, a precipitação para o período foi de $532,7 \mathrm{~mm}$, e umidade relativa média foi de $81,2 \%$ (Figura 1). Combinação de altos volumes de chuvas, alta umidade relativa são fatores que favorecem o aparecimento da doença, principalmente na primavera e verão para a B. cinerea (De Bem et al, 2015), além disso, para desenvolvimento da doença tem-se a faixa de $15-23^{\circ} \mathrm{C}$, como temperatura ótima para desenvolvimento da doença (Hed et al., 2009), sendo assim, durante o período de avaliação observou-se condições favoráveis a ocorrência da doença.

Figura 1. Precipitação pluviométrica acumulada $(\mathrm{mm})$, umidade relativa do ar $(\%)$ e temperatura média do ar $\left({ }^{\circ} \mathrm{C}\right)$ para São Joaquim/SC durante as safras 2016/2017.

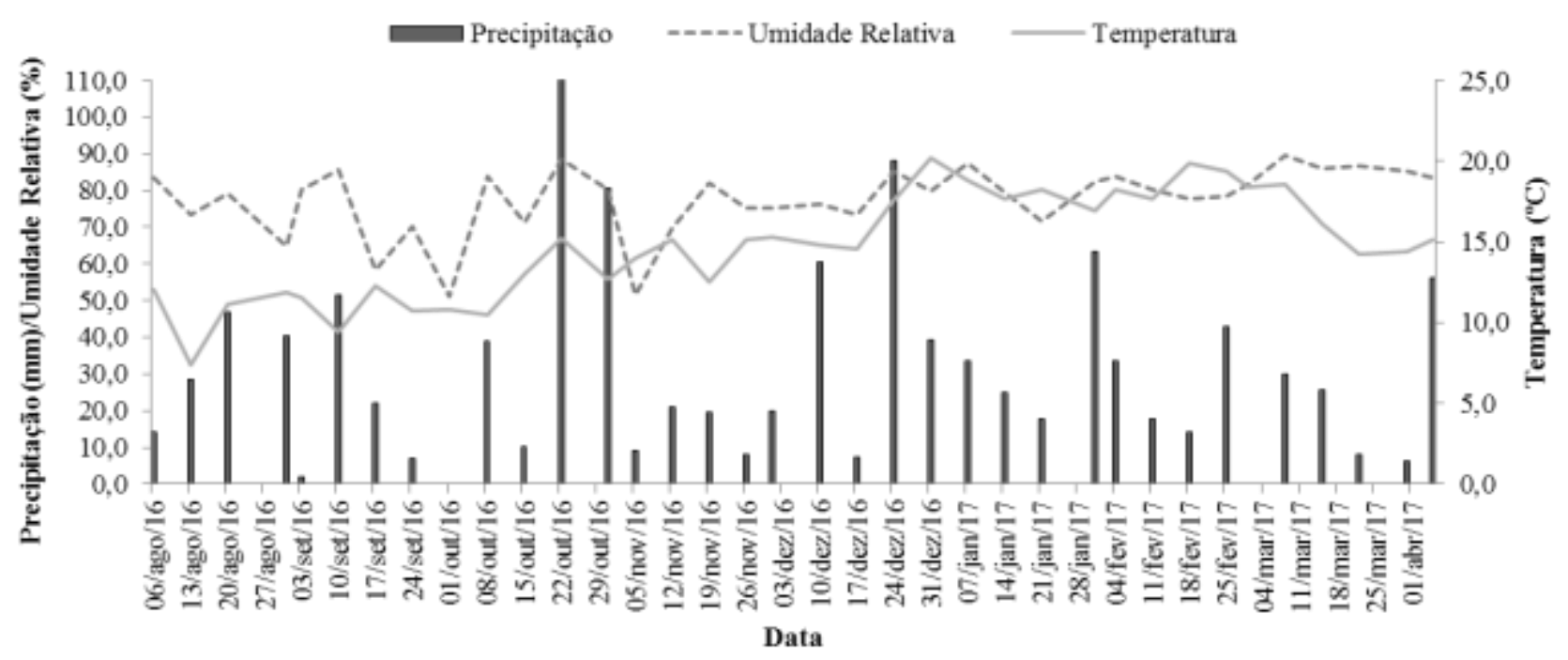

Fonte: Autores.

Observou-se efeito da carga de gemas planta ${ }^{-1}$ nas variáveis epidemiológicas da podridão cinzenta nos cachos da videira Cabernet Franc. O início do aparecimento dos sintomas ocorreu aos 26 dias para a carga de 15 gemas planta $^{-1}$, enquanto para as cargas de 30, 50 e 75 gemas planta $^{-1}$ ocorreram aos 17, 14 e 12 dias, respectivamente, não diferindo estatisticamente entre si (Tabela 1). Não se observou efeito da carga de gemas planta ${ }^{-1}$ para as variáveis tempo para atingir a máxima incidência e severidade da doença, onde observou-se, que aos 49 dias após a primeira avaliação, os valores foram superiores para as variáveis incidência e severidade da podridão cinzenta para todos os tratamentos avaliados.

Em relação a variável início do aparecimento dos sintomas, deve-se considerar que Botrytis cinerea sobrevive no solo na forma de micélio em restos culturais e gemas, e na forma de escleródios na casca do ramo. Frutos mumificados da safra 
anterior também proporcionam substrato para sua sobrevivência (Garrido \& Sônego, 2005). Nesse contexto, com aumento da carga de gemas planta ${ }^{-1}$, tem-se maior número de estruturas deixadas no momento da poda, e por consequência, pode haver maior fonte de inóculo em plantas submetidas a maiores cargas de gemas planta ${ }^{-1}$, podendo influenciar o início da epidemia no vinhedo.

A incidência de podridão cinzenta nos cachos foi superior à medida que se aumentava a carga de gemas planta ${ }^{-1}$. A carga de 15 gemas planta $^{-1}$ apesentou incidência da podridão cinzenta de 73,4\%, enquanto as cargas de 30, 50 e 75 apresentam incidência de 85,9, 95,3 e 100,0\%. A severidade da podridão cinzenta foi superior na carga de 75 gemas planta $^{-1}$, com valor de $7,9 \%$, enquanto a carga de 15 gemas planta $^{-1}$ apresentou a menor severidade da doença com 1,9, e as cargas de 30 e 50 gemas planta $^{-1}$ apresentaram valores intermediários aos demais, com 4,4 e 6,5\% de severidade da doença, respectivamente.

Observou-se que o aumento da carga de gemas planta $^{-1}$ resultou em aumento da intensidade da podridão cinzenta, $o$ que pode ser explicado pelo microclima favorável que ocorre pelo adensamento dos cachos, pois de acordo com Mossetti et al. (2016) o desenvolvimento de $B$. cinerea é fortemente influenciado pelo microclima na zona dos cachos

Trabalhos realizados por Greven et al., 2014 e Wurz et al. (2020a), demonstraram que o aumento da carga de gemas planta-1, resulta em maior número de cachos planta $^{-1}$. Trabalho realizado por Wurz et al. (2017a) e Wurz et al. (2019), avaliando o efeito da carga de gemas planta ${ }^{-1}$ na ocorrência de antracnose em ramos e folhas da videira Cabernet Franc, observaram que acima cargas acima de 50 gemas lanta $^{-1}$ resultam em maiores valores de doença.

Para a variável AACPID, observou-se que as cargas de 50 e 75 gemas planta $^{-1}$ apresentaram os maiores valores, com 1585,9 e 1667,9, respectivamente, enquanto a carga de 30 gemas planta $^{-1}$ apresentou AACPID de 1194,6, e o menor valor de AACPID foi observado na carga de 15 gemas planta $^{-1}$, com 760,1 (Tabela 1). A AACPSD apresentou comportamento similar, com os maiores valores sendo observados nas cargas de 50 e 75 gemas planta $^{-1}$, apresentando valores de 54,7 e 63,4 , respectivamente, e o menor valor na carga de 15 gemas planta $^{-1}$, com valor de 15,0.

De acordo com O'Daniel et al. (2012), o aumento da carga de gemas planta ${ }^{-1}$ resulta em aumento do número de ramos por hectare, e consequentemente uma diminuição do espaçamento entre ramos. Essas repostas indicam um dossel mais denso, permitindo menor penetração da radiação solar, redução da eficiência dos tratamentos fitossanitários e redução do fluxo de ar no dossel vegetativo, criando um microclima com elevada umidade, podendo influenciar na ocorrência de doenças fúngicas e qualidade dos cachos (Smart, 1985).

Tabela 1. Efeito da carga de gemas no início do aparecimento dos sintomas (IAS), tempo para atingir a máxima incidência (TAMISD) e severidade (TAMSD) da podridão cinzenta, incidência máxima (Imax), severidade máxima (Smax), e área abaixa da curva de progresso da incidência (AACPID) e severidade (AACPSD) da podridão cinzenta na videira Cabernet Franc (Vitis vinifera L.) em região de elevada altitude de Santa Catarina. Safra 2017.

\begin{tabular}{|c|c|c|c|c|c|}
\hline & \multicolumn{4}{|c|}{ Carga de Gemas } & \multirow{2}{*}{$\frac{\mathrm{CV}}{(\%)}$} \\
\hline & 15 & 30 & 50 & 75 & \\
\hline \multicolumn{6}{|l|}{2017} \\
\hline IAS (dias) & $26 \mathrm{a}$ & $17 \mathrm{~b}$ & $14 \mathrm{~b}$ & $12 \mathrm{~b}$ & 22,1 \\
\hline TAMID (dias) & $49 \mathrm{~ns}$ & 49 & 49 & 49 & 0,0 \\
\hline TAMSD (dias) & $49 \mathrm{~ns}$ & 49 & 49 & 49 & 0,0 \\
\hline Incidência máxima (\%) & $73,4 \mathrm{~b}$ & $85,9 \mathrm{ab}$ & $95,3 \mathrm{a}$ & $100,0 \mathrm{a}$ & 10,3 \\
\hline Severidade máxima (\%) & $1,9 \mathrm{c}$ & $4,4 \mathrm{bc}$ & $6,5 a b$ & 7,9 a & 26,4 \\
\hline AACPID & $760,1 \mathrm{c}$ & $1197,6 \mathrm{~b}$ & $1585,9 \mathrm{a}$ & $1667,9 \mathrm{a}$ & 11,1 \\
\hline AACPSD & $15,0 \mathrm{c}$ & $34,8 \mathrm{bc}$ & $54,7 \mathrm{ab}$ & $63,4 \mathrm{a}$ & 22,0 \\
\hline
\end{tabular}

*Médias seguidas da mesma letra, na linha, não diferem entre si pelo teste Tukey a $5 \%$ de probabilidade de erro.ns = não significativo pela análise de variância (ANOVA) a 5\% de probabilidade de erro. Fonte: Autores. 
A primeira avaliação da podridão cinzenta ocorreu dia $02 / f e v$, correspondendo ao início do veráison. Para a variável AACPID observou-se aumento gradual dessa variável até o dia 02/mar, e após esse período observou-se aumento acentuado da AACPID, com destaque para as cargas de 50 e 75 gemas planta $^{-1}$, que apresentaram valores superiores em relação as cargas de 15 e 30 gemas planta $^{-1}$ (Figura 2). Observou-se aumento da AACPID até o momento da colheita.

Figura 2. Efeito da carga de gemas na área abaixo da curva de progresso da incidência da podridão cinzenta nos cachos da videira Cabernet Franc (Vitis vinifera L.) em região de elevada altitude de Santa Catarina. Safra 2017.

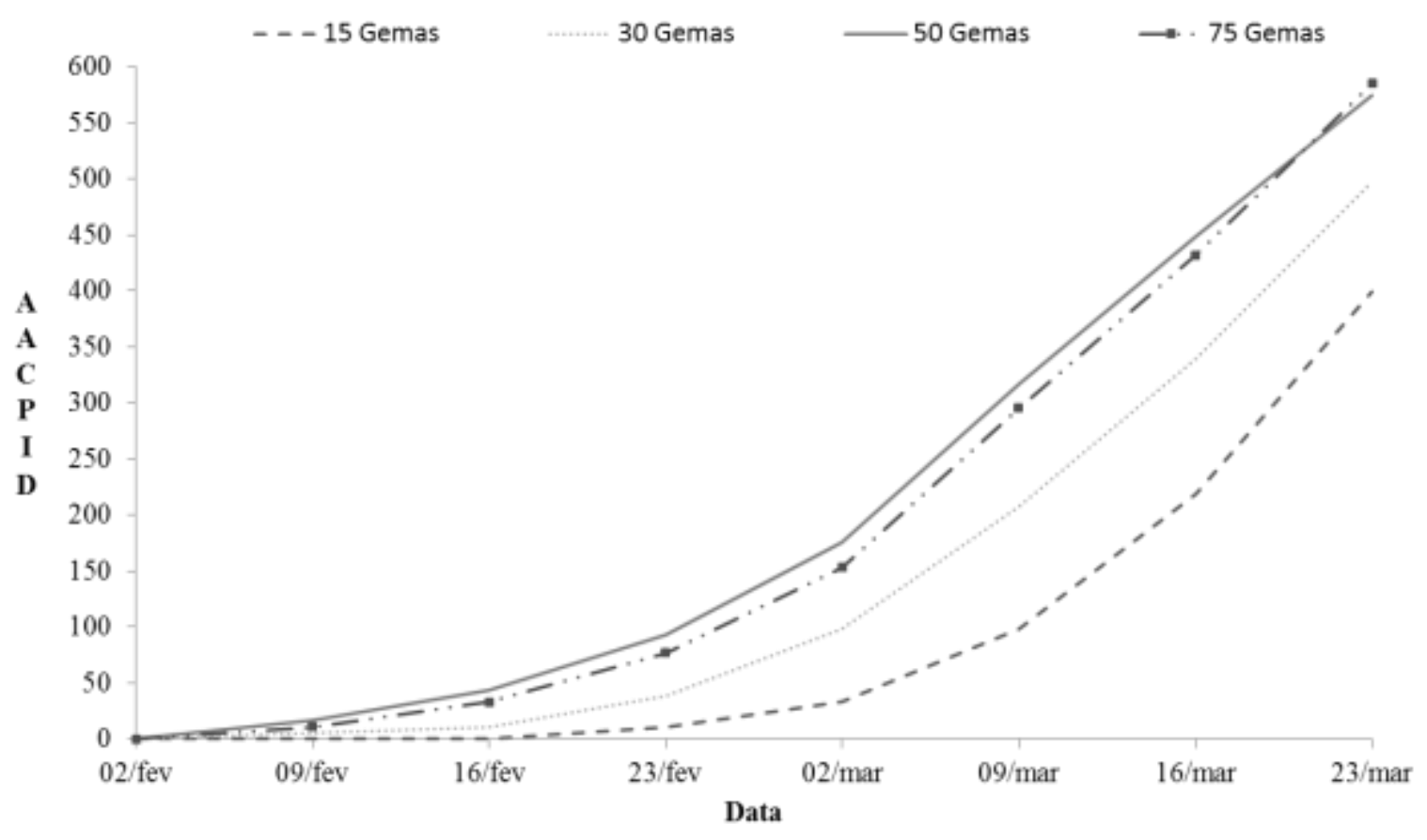

Fonte: Autores.

A variável AACPSD apresentou comportamento semelhante a AACPID. Até o dia 02/mar, o aumento do valor da AACPSD pode ser considerado baixo. Contudo após o dia 02/mar, observou-se aumento significativo para as cargas de 50 e 75 gemas planta ${ }^{-1}$ (Figura 3). A carga de 15 gemas planta $^{-1}$ apresentou aumento baixo e constante até o dia 16/mar, e após esse período observou-se acréscimo mais acentuado, no entanto, para as demais cargas de gemas planta ${ }^{-1}$, observou-se que o aumento dos valores AACPSD, ocorreu anteriormente ao dia 16/mar, apresentando efeito da carga de gemas na AACPD, com os maiores valores sendo observados nas cargas de 50 e 75 gemas planta $^{-1}$.

As curvas de progresso da doença, usualmente expressas pela plotagem da proporção de doença versus tempo, é a melhor representação de uma epidemia. Por meio dela, pode-se caracterizar interações entre patógeno, hospedeiro e ambiente sendo possível criar estratégias de controle e prever níveis futuros de doença. (Bergamin Filho, 2011). Houve diferenças significativas entre as cargas de gemas planta ${ }^{-1}$ em relação a área abaixo da curva de progresso da incidência e severidade da doença (AACPID e AACPSD), indicando portanto, que o aumento da carga de gemas planta ${ }^{-1}$ resultou em uma maior suscetibilidade a ocorrência da podridão cinzenta nos cachos da videira Cabernet Franc. 
Figura 3. Efeito da carga de gemas na área abaixo da curva de progresso da severidade da podridão cinzenta nos cachos da videira Cabernet Franc (Vitis vinifera L.) em região de elevada altitude de Santa Catarina. Safra 2017.

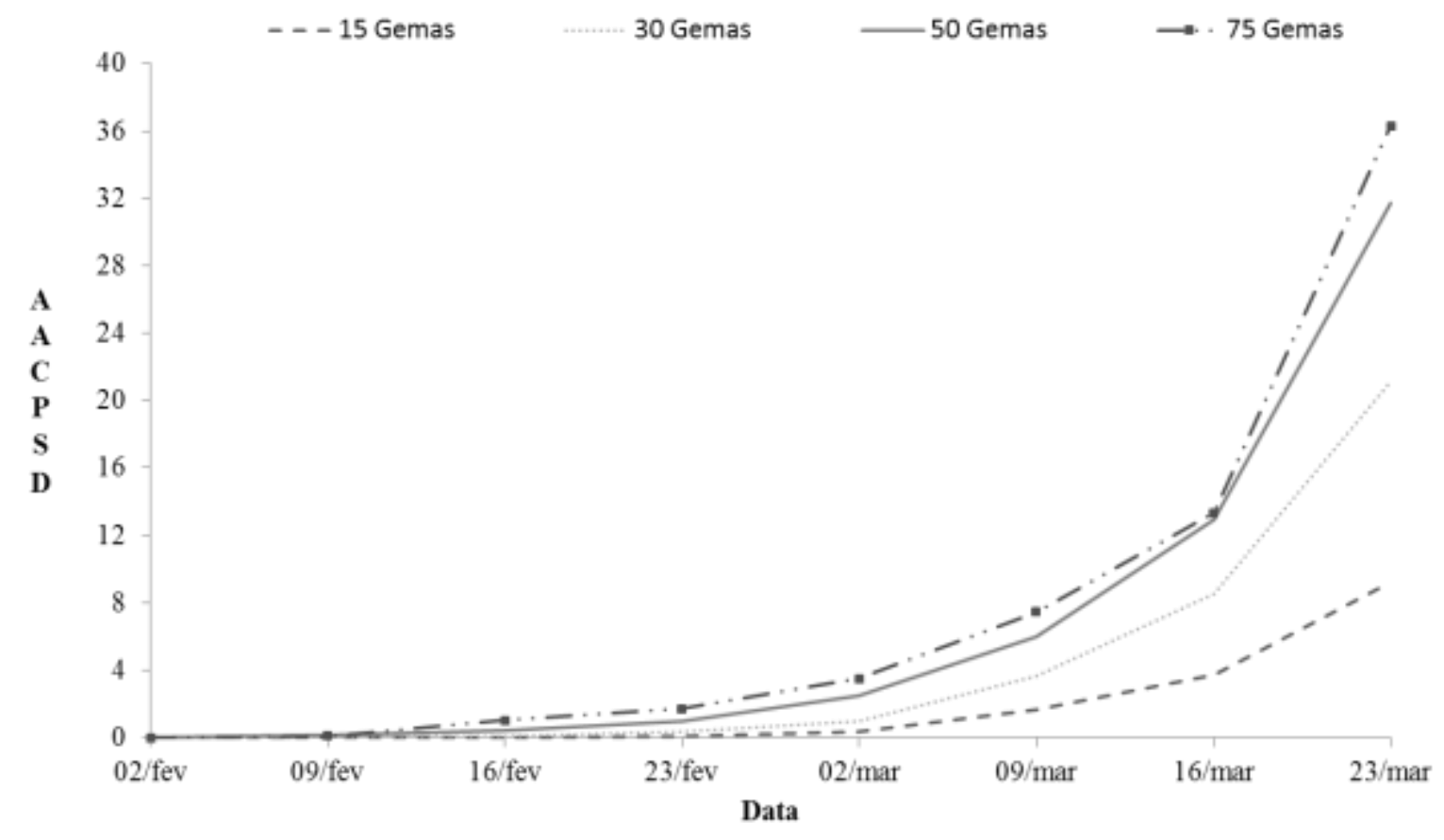

Fonte: Autores.

Um dos principais fatores que limitam a produção de uva é a ocorrência da podridão cinzenta ou podridão de Botrytis causada pelo fungo Botryotinia fuckeliana (de Bary) Whetzel, forma sexuada de Botrytis cinerea Persoon ex Fries (Ellis, 1971), portanto, a adoção de uma elevada carga de gemas planta $^{-1}$, deve ser acompanhada de uma série de medidas preventivas, com objetivo de reduzir os danos causados pela podridão cinzenta.

De acordo com Molitor et al. (2012), Molitor et al. (2014) e Bem et al. (2017), medidas culturais que resultam em maior exposição solar e ventilação na região dos cachos, como o manejo da desfolha, são ferramentas eficientes nas estratégias de controle da $B$. cinerea nos cachos da videira. E em plantas submetidas a elevadas cargas de gemas planta ${ }^{-1}$, deve-se realizar essas medidas culturais, como a desfolha no estádio baga chumbinho, proporcionando maio eficiência no controle da doença (Wurz et al., 2017b; Wurz et al., 2020b).

\section{Conclusão}

1. O aumento da carga de gemas não influenciou o tempo para atingir a máxima incidência e severidade da doença.

2. O início do aparecimento dos sintomas foi mais precoce para as plantas submetidas aos níveis de poda de 30,50 e 75 gemas planta $^{-1}$.

3. O aumento da carga de gemas resultou em aumento da incidência, severidade, área abaixo da curva de progresso da incidência e severidade da doença, sendo os maiores valores observados nas plantas submetidas a níveis de poda de 50 e 75 gemas planta ${ }^{-1}$.

4. A adoção de elevado número de gemas planta ${ }^{-1}$, deve ser acompanhada de práticas de manejo integrado de doenças que visam reduzir os efeitos ocasionados pela ocorrência de podridão cinzenta. Novos trabalhos devem ser conduzidos relacionando práticas de manejo do dossel vegetativo com o aumento da carga de gemas planta ${ }^{-1}$. 


\section{Referências}

Bergamin Filho, A. (2011) Curvas de progresso da doença. In: Bergamin Filho, A., Kimati, H., Amorin, L. (Eds.), Manual de fitopatologia, São Paulo: 647666.

Campbell, C. L. \& Madden, L. V (1990). Introduction to plant disease epidemiology. Wiley, p. 560.

Clingeleffer, P. R (2009) Influence of canopy management systems on vine productivity and fruit composition. In: Recent Advances in Grapevine Canopy Management. University of California. p.13-19.

De Bem, B. P., Bogo, A., Everhart, S., Casa, R. T., Gonçalves, M. J., Marcon Filho, J. L. \& Cunha, I. C. (2015). Effect of Y-trellis and Vertical Shoot Positioning Training Systems on Downy Mildew and Botrytis Bunch Rot of Grape in Highlands of Southern Brazil. Scientia Horticulturae, $185,162-166$.

De Bem, B. P., Bogo, A., Allebrandt, R., Marcon Filho, J. L., Wurz, D. A., Brighenti, A. F. \& Rufato, L. (2017). Botrytis bunch rot on 'Sauvignon Blanc' grapevine on the Y-trellis and vertical shoot-positioned training systems. Pesquisa Agropecuária Brasileira, 52, 818-822.

Ellis, M. B. (1971). Dematiaceous Hyphomycetes. Commonwealth Mycological Institute, Kew.

Garrido, L. R \& Sônego, O. R. (2005). Podridão Cinzenta da uva: epidemiologia, sintomatologia e controle. Bento Gonçalves: Embrapa Uva e Vinho, 07p. Circular Técnica 59, 07p.

Greven, M. M., Bennett, J. S. \& Neal, S. M. (2014). Influence of retained node number on Sauvignon Blanc grapevine vegetative growth and yield. Australian Journal of Grape and Wine Research, 20(2), 263-271.

Greven, M. M., Neal, S. M. \& Bennett, J. S. (2015). Influence of retained node number on Sauvignon Blanc grapevine phenology in a cool climate. Australian Journal of Grape and Wine Research, 21(2), 209-301.

Hed, B., Ngugi, H. K. \& Travis, J. W. (2009). Relationship between cluster compactness and bunch rot in vignoles grapes. Plant Disease, 93(11), 1195-1201.

Hill, G. N., Beresford, R. M. \& Evans, K. J. (2010). Tools for accurate assessment of botrytis bunch rot (Botrytis cinerea) on wine grapes. New Zealand Plant Protection, 63, 174-181.

Miele, A. \& Mandelli, F. (2012). Manejo do dossel vegetativo e seu efeito nos componentes de produção da videira Merlot. Revista Brasileira de Fruticultura, 34, 964-973.

Miele, A. \& Rizzon, L. A. (2013). Intensidades da poda seca e do desbaste de cacho na composição da uva Cabernet Sauvignon. Revista Brasileira de Fruticultura, 35, 1081-1092.

Molitor, D., Behr, M., Hoffmann, L. \& Evers, D. (2012). Impact of grape cluster division on cluster morphology and bunch rot epidemic. American Journal of Enology and Viticulture, 63(4), 508-514.

Molitor, D., Baron, N., Sauerwein, T., André, C., Kicherer, A., Döring, J., Stoll, M., Beyer, M., Hoffmann, L. \& Evers, D. (2014). Postponing first shoot topping reduces grape cluster compactness and delays bunch rot epidemic. American Journal of Enology and Viticulture, 66, 164-176.

Mossetti, D., Herrera, J.C., Sabbatini, P., Green, A., Alberti, G., Peterlunger, E., Lisjak, K. \& Catellarin, S. D. (2016). Impact of leaf removal after berry set on fruit composition and bunch rot in 'Sauvignon blanc'. Vitis, 55, 57-64.

O’Daniel, S. B., Archbold, D. D. \& Kurtural, S. K. (2012). Effects of balanced pruning severity on Traminette (Vitis spp.) in a warm climate. American Journal of Enology and Viticulture, 63(2), 284-290.

Santos, H. G., Jacomine, P. K., Anjos, L. H., Oliveira, V. A., Lumbreras, J. F., Coelho, M. R., Almeida, J. A., Araujo Filho, J. C., Oliveira, J. B. \& Cunha, T. J. (2018). Sistema Brasileira de Classificação do Solo, Embrapa, 356p.

Smart, R. E. (1985). Principles of grapevine canopy microclimate manipulation with implications for yield and quality. A Review. American Journal of Enology and Viticulture, 36(3), 230-239.

Tonietto, J. \& Carbonneau, A. (2004). A multicriteria climatic classification system for grape-growing regions worlwide. Agricultural and Forest Meteorology, 124, 81-97.

Wurz, D. A., Allebrandt, R., Bem, B. P., Brighenti, A. F., Reinehr, J., Canossa, A. T., Bonin, B. F., Kretzschmar, A. A. \& Rufato, L. (2017a) Ocorrência de antracnose nas videiras Sauvignon Blanc e Cabernet Franc em função da carga de gemas. Revista Brasileira de Viticultura e Enologia, 9, $42-46$.

Würz, D. A., Brighenti, A. F., Allebrandt, R., Marcon Filho, J. L., Bem, B. P., Araújo Filho, J. V., Rufato, L. \& Kretzschmar, A. A. (2017b). Desfolha precoce como estratégia de controle da podridão de Botrytis cinerea na videira Cabernet Sauvignon em regiões de altitude. Summa Phytopathologica, 43 , 111-117.

Wurz, D. A., Bonin, B. F., Allebrandt, R., Bem, B. P., Canossa, A. T., Reinher, J., Rufato, L., Brighenti, A. F. \& Kretzschmar, A. A. (2019). Efeito da carga de gemas da videira 'Sauvignon blanc' na intensidade de antracnose (Elsinoe ampelina). Revista Eletrônica Científica UERGS, 5(1), 75-78.

Wurz, D. A., Bonin, B. F., Brighenti, A. F., Canossa, A. T., Reinher, J., Allebrandt, R., Bem, B. P., Rufato, R. \& Kretzschmar, A. A. (2020a). Maior carga de gemas da videira resulta em melhora dos índices produtivos e vegetativos da videira 'Cabernet Franc' cultivada em região de elevada altitude. Revista de Ciências Agroveterinárias, 19(2), 171-177.

Würz, D. A., Rufato, L., Bogo, A., Allebrandt, R., Bem, B. P., Marcon Filho, J. L., Brighenti, A. F. \& Bonin, B. F. (2020b). Effects of leaf removal on grape cluster architecture and control of Botrytis bunch rot in Sauvignon Blanc grapevines in Southern Brazil. Crop Protection, 131 , e-105079.

Zoecklein, B. W., Wolf, T. K., Duncan, N. W., Judge, J. M. \& Cook, M. K. (1992). Effects of fruit zone leaf removal on yield, fruit composition, and fruit rot incidence of Chardonnay and White riesling (Vitis vinifera L.) grapes. American Journal of Enology and Viticulture, 43, 139-148. 